\section{Vereinbarkeit von Familie und Beruf Faktor für berufliche Zufriedenheit}

Bei der Wahl des künftigen Arbeitgebers sind auch für Ärztinnen und Ärzte schon lange nicht mehr nur das Gehalt und die Karrieremöglichkeiten ausschlaggebend. Mindestens ebenso wichtig ist es, dass sich Familie, Freizeit und Beruf unter einen Hut bringen lassen. Dies gilt sowohl für die Niederlassung in einer Praxis als auch für die Arbeit im Krankenhaus. Auf einer gemeinsam von der Landesärztekammer Rheinland-Pfalz, der Bundesarbeitsgemeinschaft Leitender Pflegepersonen sowie dem Verband der Krankenhausdirektoren veranstalteten Fachtagung in Mainz diskutierten Experten aus dem Gesundheitswesen darüber, wie sich betriebliche Anforderungen und individuelle Lebenskonzepte in Einklang bringen lassen.

Viele Krankenhausärzte fühlen sich in ihrem Beruf nicht wohl. Einer Befragung von 729 Assistenzärzten durch die Fachhochschule Münster zufolge sind $20 \%$ mit ihrer Berufswahl unzufrieden. Nur $40 \%$ können sich mit ihrer Arbeitsstelle identifizieren. Lediglich ein Drittel würde den Arztberuf grundsätzlich weiterempfehlen.

Hauptgründe für die Unzufriedenheit sind Stress im Berufsalltag (57,2\%) und zu wenig Freizeit $(54,6 \%)$ beziehungsweise eine fehlende Bezahlung von Überstunden oder die Möglichkeit zum Freizeitausgleich (49,6\%). Neben einem guten Betriebsklima wünschen sich 94,7\% der befragten Assistenzärzte eine bessere Vereinbarkeit von Beruf und Familie.

\section{Flexiblere Arbeitszeiten und ver- lässliche Kinderbetreuung sind wichtig}

„Familienfreundlichkeit fordert in den nächsten Jahren Praxen und Krankenhäuser noch intensiver als bisher heraus. Die Nachfrage nach familienfreundlichen Arbeitszeiten und nach mehr Teilzeitarbeitsstellen für Ärzte und Ärztinnen wird weiter zunehmen“, so Prof. Frieder Hessenauer, Präsident der Landesärztekammer Rheinland-Pfalz (LÄK RP) bei einer Fortbildungsveranstaltung in Mainz.

Flexiblere Arbeitszeitmodelle und ver-

lässliche Kinderbetreuungen seien wichtig, damit Ärztinnen und Ärzte sich

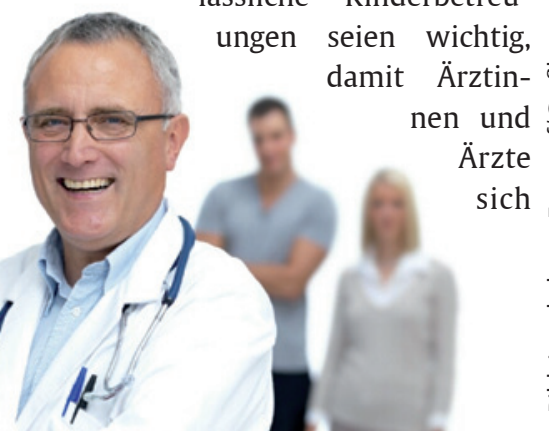

nicht zwischen Beruf und Familie zerreißen und verschleißen, sondern auch in Zukunft ihren Traumberuf gut, kompetent, verlässlich und vor allem zufrieden ausüben können, betonte Hessenauer.

Dies gelte auch für das Pflegepersonal, ergänzte Doris Seis von der Bundesarbeitsgemeinschaft Leitender Pflegepersonen. Seis forderte mehr kreative Ansätze und eine stärke Kooperation der Beteiligten, einschließlich der Politik. Eine Mitarbeiterbindung werde viel zu häufig noch reaktiv betrieben, wenn eine Fachkraft zum Beispiel kündigen wolle, kritisierte Konrad Einig, Leiter Personalmanagement im Koblenzer Stiftungsklinikum Mittelrhein. An deutschen Krankenhäusern sind ferner Angebote zur Kinderbetreuung bislang Mangelware. Eine Studie des Deutschen Krankenhausinstituts ergab, dass lediglich 19,2\% aller Kliniken eine betriebliche Kinderbetreuung vorhalten.

Wie bedeutsam ein Betriebskindergarten für die Personalgewinnung und -bindung sein kann, belegt indes das Beispiel des DRK-Krankenhauses Kirchen. Bereits in den Vorstellungsgesprächen werde auf die Möglichkeit hingewiesen, den Nachwuchs während der Arbeitszeiten im Kindergarten des Krankenhauses unterbringen zu können, machte Klaus Schmidt, Kaufmännischer Direktor der Klinik, deutlich. „Insbesondere beim ärztlichen Dienst stößt das Angebot auf großes Interesse.“ Dies habe im Ergebnis zu einer Zunahme der Bewerbungen und eine höhere Personalbindung geführt, so Schmidt.

Flexible Arbeitszeitangebote sind auch deshalb von enormer Bedeutung, da es unter anderem vor dem Hintergrund der demografischen Entwicklung aller Voraussicht nach in den nächsten Jahren zu 흐 einem dramatischen Fachkräftemangel kommen wird. Dr. Annegret Schoeller von der Bundesärztekammer (BÄK) wies darauf hin, dass Studien zufolge die Personallücke im ärztlichen Bereich bis 2030 schätzungsweise auf 165000 Kräfte anwachsen wird. Im nicht-ärztlichen Bereich blieben zum selben Zeitpunkt möglicherweise sogar 786000 Stellen unbesetzt.

„Bislang ist Teilzeitarbeit die häufigste Antwort auf die Doppelbelastung durch Arbeit und familiäre Pflichten“, sagte Schoeller. „Sinnvoller wäre jedoch der Ausbau von Betreuungsmöglichkeiten, um die Teilzeitarbeit zurückzudrängen und damit das Fachkräftepotenzial insbesondere bei Frauen besser auszuschöpfen.“

\section{Familienfreundlichkeit als Erfolgsfaktor im Wettbewerb}

Familienfreundlichkeit könne zudem ein wichtiger Erfolgsfaktor im Wettbewerb und die besten Mitarbeiterinnen und Mitarbeiter auf dem Arbeitsmarkt sein, betonte Bernd Decker vom Verband der Krankenhausdirektoren. Dabei konkurrieren Krankenhäuser sowohl untereinander als auch mit niedergelassenen Praxen um qualifiziertes Personal.

Eine geringere Fluktuationsrate und eine höhere Zufriedenheit rechneten sich auch betriebswirtschaftlich für Krankenhäuser und andere Gesundheitseinrichtungen, ist die Gesundheitsministerin von Rheinland-Pfalz Malu Dreyer überzeugt. Sinnvolle Lösungsansätze, die mehr Flexibilität ermöglichten, seien beispielsweise Weiterbildungsangebote in Teilzeit, Telearbeitsplätze sowie Fortbildungsmöglichkeiten während der Elternzeit beziehungsweise Beratungsangebote für Wiedereinsteiger in den Beruf. Um familienfreundliche Angebote im Gesundheitswesen flächendeckend realisieren zu können, bedürfe es der gemeinsamen Anstrengung von Politik, Unternehmen und der ärztlichen Selbstverwaltung, mahnte Schoeller. Andere europäische Staaten, wie Frankreich und die skandinavischen Länder, seien hier schon weiter.

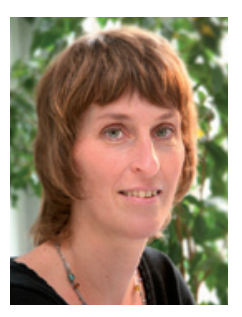

Korrespondenz Petra Spielberg Fachjournalistin für Gesundheits- und Sozialpolitik Wiesbaden/Brüssel Fax. 0611/98 818512 p.spielberg@t-online.de 